\title{
Debridamento Foraminal no Protocolo de Atendimento de Endodontistas
}

\section{Foramen Debridement in the Protocol of Attendance of Endodontists}

\section{Rosany Laurentina Santos de Carvalho ${ }^{1}$, Carolina dos Santos Guimaraes ${ }^{1}$, Roberto Alves dos Santos ${ }^{2}$, Jose Thadeu Pinheiro ${ }^{3}$}

\begin{abstract}
Introduction: Endodontics suffered the influence of a variety of concepts and philosophies that characterized the different periods of its evolution. Foramen Debridement is an endodontic procedure that aims the passive cleaness of cementary channel and can be considered as a pertinent example of controversial topics in endodontics. Objective: To evaluate the attitudes of endodontists in making the foramen debridement of necrotic pulp in cases with and without periapical lesion and which moment they consider convenient to carry out this surgical maneuver. Materials and methods: The study was conducted through a questionnaire to evaluate the knowledge of endodontists of Pernambuco Brazil, about foramen debridement. In this questionnaire could be found general information about identification and specific of foramen debridement procedure and its realization during biomechanical preparation of root canals diagnosed with pulp necrosis with or without periapical lesion radiographically visible. Results: The response rate, a sample of 115 professionals, was $63.5 \%$. Most experts had over 20 years of graduates $(49.5 \%)$ and when asked if they perform foramen debridement d $97.3 \%$ responded yes. Regarding the clinical situation in which perform the foramen debridement only $25.3 \%$ answered at all endodontic treatments and $54.9 \%$ in all cases necrosis. Conclusion: The results of this research suggest that foramen debridement Consolidated protocols endodontic specialists of Pernambuco.

Descriptors: Endodontics; debridement; decontamination
\end{abstract}

\section{Resumo}

Introdução: A Endodontia sofreu a influencia de uma variedade de conceitos e filosofias, que caracterizaram os diferentes períodos da sua evolução. O desbridamento do forame é um procedimento endodôntico que visa à limpeza passiva do canal cementário e pode ser considerado como um exemplo pertinente de temas controversos na endodontia. Objetivo: avaliar as atitudes dos endodontistas na realização do desbridamento foraminal nos casos de necrose pulpar com e sem lesão periapical e qual momento consideram oportuno para a realização desta manobra operatória. Materiais e métodos: A pesquisa foi realizada através da aplicação de um questionário para verificar o conhecimento dos endodontistas de Pernambuco Brasil, acerca do desbridamento foraminal. No questionário constatavam informações gerais de identificação e específicas do procedimento de desbridamento foraminal e da sua realização durante o preparo biomecânico de canais radiculares diagnosticados com Necrose pulpar com ou sem lesão periapical visível radiograficamente. Resultados: A taxa de resposta, de uma amostra de 115 profissionais, foi de $63,5 \%$. A maioria dos especialistas tinha mais de 20 anos de formados $(49,5 \%)$ e quando perguntados se realizam o desbridamento foraminal $97,3 \%$ responderam que sim. Quanto à situação clínica em que realizam o desbridamento foraminal apenas $25,3 \%$ responderam em todos os o tratamentos endodônticos e $54,9 \%$ em todos os casos de necrose. Conclusão: desbridamento foraminal está consolidado nos protocolos endodônticos de especialistas de Pernambuco.

Descritores: Endodontia; desbridamento; descontaminação

\begin{abstract}
1 Doutoranda do Curso de Pós graduação em Endodontia da Faculdade de Odontologia de Pernambuco Universidade de Pernambuco - UPE Camaragibe, Pernambuco - Brasil.

${ }^{2}$ Coordenador Departamento de Pós-graduação em Endodontia da Faculdade de Odontologia de Pernambuco Universidade de Pernambuco - UPE Camaragibe, Pernambuco - Brasil.

3 Professor Titular da Disciplina de Endodontia da Universidade Federal de Pernambuco e Prof. Adjunto da Disciplina de Endodontia da Universidade de Pernambuco.
\end{abstract}

Correspondência: Rosany Laurentina Santos de Carvalho

Endereço: Rua Dr Osvaldo Salsa, 100 / 1501, Graças - CEP 52011-170 - Recife Pernambuco - Brasil

Fone: (81) 88473558 / 32213556

Email: rosanycarvalho@hotmail.com

Data de Submissão: 30/10/2012

Data de Aceite: 03/07/2013

\section{Introdução}

A Endodontia ao longo do tempo sofreu a influencia de uma variedade de conceitos e filosofias, que caracterizaram os diferentes períodos da sua evolução (SILVA; GUIMARAES; SILVEIRA, 2009)

Entretanto, apesar dos recentes avanços, muitos procedimentos ainda são amplamente debatidos entre os profissionais, necessitando, portanto de mais pesquisas para serem amplamente aceitos e utilizados. A influência do desbridamento foraminal na resolução de alterações necróticas endodônticas pode ser considerada como um exemplo pertinente de temas controversos na endodontia (GUIMARÃES; SILVA; SANTOS, 2009; SOUZA, 2006)

O desbridamento do forame é um procedimento que se incorpora a ação mecânica da instrumentação e química das soluções irrigadoras e medicação intracanal. Visa através da limpeza passiva do canal cementário, com instrumentos de pequeno calibre, eliminar a infecção estabelecida neste (SOUZA, 2006), entretanto, esta manobra constitui apenas um passo a mais na busca da limpeza foraminal e por si só não será capaz de levar o tratamento ao sucesso (GUIMARÃES; SILVA; SANTOS, 2009).

Diante do risco de traumas indesejáveis e desnecessários para os tecidos periapicais, alguns estudos defendem que 0 tratamento endodôntico só deve ser realizado no canal dentinário em um comprimento de trabalho localizado a $1 \mathrm{~mm}$ do ápice radiográfico (SOUZA, 2006). No entanto, a extensão do canal, com um diâmetro de aproximadamente 0,25 milímetros, tem espaço suficiente para abrigar quase 80.000 microorganismos (SOUZA, 2006) com absoluta prevalência de bactérias anaeróbicas (LEONARDI; ATLAS; RAINDEN, 2007). Portanto, foi postulado que a limpeza dos canais deve estender-se ao canal cementário, promovendo assim a limpeza do forame apical (SCHAEFFER; WHITE; WALTON, 2005; GUIMARÃES, 2006)

A patência apical e o tipo de material obturador de canal influenciam diretamente no processo de reparo apical (CAMOES; SALLES; FERNANDO et al., 2009). O emprego de um cimento a base de hidróxido de cálcio em dentes sem a realização da patência

Rev. Fac. Odontol. Porto Alegre, v. 53, n. 2, p. 15-18, mai./ago., 2012. 
apical pode promover melhores resultados (SILVA; CRUZ; ARAUJO; PESSOA, 2009).

Por outro lado, a literatura mostra que todas as técnicas de preparo biomecânico podem causar extrusão periapical (RIBEIRO; MALNATI; COSTA JÚNIOR, 2010). Entretanto o tamanho da preparação apical final, observando-se aspectos como forma e diâmetro da constrição apical, anatomia radicular, técnica de preparo biomecânico manual ou rotatória, com aumento de patência foraminal, reduz a contagem de bactérias e resulta em preparos apicais mais amplos com canais mais limpos (TINAZ; ALACAM; UZUN et al.; 2005; BAUGH; WALLACE, 2005).

Guimarães (2006) avaliou o efeito de desbridamento foraminal na reparação de lesões periapicais detectados por radiografia. Uma amostra composta por 980 dentes mostrando lesões periapicais visíveis radiograficamente foi submetida a tratamento endodôntico, onde 402 tratamentos endodônticos foram realizados sem o procedimento do desbridamento foraminal e os 578 dentes restantes tiveram os tratamentos endodônticos realizados com desbridamento. Após o intervalo de um ano, os dentes foram submetidos à avaliação clínica e radiográfica. Os resultados mostraram não haver diferença estatisticamente significativa entre os valores encontrados nos dois grupos, sugerindo que o desbridamento do forame não é um fator determinante para a reparação de lesões periapicais.

Portanto, os objetivos deste estudo foram avaliar as atitudes dos endodontistas na realização do desbridamento foraminal nos casos de necrose pulpar com e sem lesão periapical e qual momento consideram oportuno para a realização desta manobra operatória.

\section{Metodologia}

Este estudo observacional transversal, com abordagem quantitativa, foi aprovado pelo Comitê de Ética em Pesquisa da Universidade de Pernambuco. A relação dos Cirurgiões-Dentistas atuantes em serviços públicos ou privados do estado de Pernambuco, inscritos na especialidade de Endodontia e com dados completos no Conselho Regional de Odontologia de Pernambuco (CRO-PE) foi incluída $(n=115)$.

A pesquisa foi realizada através da aplicação de um questionário (Figura 1), para verificar o conhecimento destes profissionais acerca do desbridamento foraminal. O questionário era composto de 10 perguntas, onde constatavam informações gerais de identificação e específicas do procedimento de desbridamento foraminal e a sua realização durante o preparo biomecânico de canais radiculares diagnosticados com Necrose pulpar com ou sem lesão periapical visível radiograficamente. Este foi enviado aos Cirurgiões-Dentistas em envelope lacrado, via correios, por mala direta do CRO-PE, com o envelope postado para devolução juntamente com duas cópias do Termo de Consentimento Livre e Esclarecido (TCLE), onde o profissional declara que aceita participar voluntariamente da pesquisa. Os questionários respondidos e devolvidos no prazo máximo de 60 dias a partir da data de postagem foram incorporados a amostra. Os dados recebidos após esse prazo foram considerados perda.

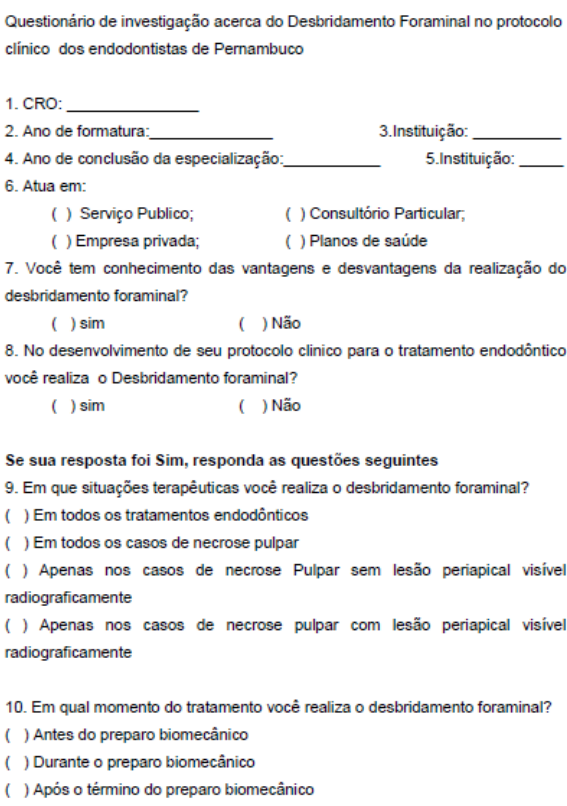

Figura 1 - Questionário para avaliação do conhecimento dos endodontistas acerca do desbridamento foraminal.

As respostas dos questionários foram tabuladas $e$ as distribuições de porcentagem para cada item foram computadas. Testes de proporção foram utilizados para analisar as variáveis categóricas ou nominais.

\section{Resultados}

Dos 115 questionários entregues, 73 foram devolvidos, com uma taxa de resposta de $63,5 \%$.

Tabela 1: Tempo de conclusão do curso de graduação em Odontologia em números absolutos e em porcentagem

\begin{tabular}{llc}
$\begin{array}{c}\text { Tempo de conclusão do curso de } \\
\text { graduação }\end{array}$ & $\begin{array}{l}\text { Número } \\
\text { absoluto }\end{array}$ & $\begin{array}{c}\text { Número } \\
\text { em } \%\end{array}$ \\
Mais de 20 anos & $\begin{array}{l}36 \\
\text { pessoas }\end{array}$ & $\mathbf{4 9 , 3 0 \%}$ \\
25 & $\begin{array}{l}25 \\
\text { pessoas } \\
12\end{array}$ & $\mathbf{3 4 , 2 0 \%}$ \\
Mais de 10 anos e menos de 20 anos & \\
Menos de 10 anos & pessoas & $\mathbf{1 6 , 4 0 \%}$ \\
\hline
\end{tabular}

Tabela 2: Tempo de conclusão do curso de especialização em endodontia em números absolutos e em porcentagem

\begin{tabular}{lcc}
\hline $\begin{array}{c}\text { Tempo de conclusão do curso de } \\
\text { especialização }\end{array}$ & $\begin{array}{c}\text { Número } \\
\text { absoluto } \\
\text { Mais de } 20 \text { anos }\end{array}$ & $\begin{array}{c}\text { Número } \\
\text { em } \%\end{array}$ \\
pessoas & $\mathbf{2 8 , 8 0 \%}$ \\
Mais de 10 anos e menos de 20 anos & $\begin{array}{c}10 \\
\text { pessoas } \\
42\end{array}$ & $\mathbf{1 3 , 6 0 \%}$ \\
Menos de 10 anos & pessoas & $\mathbf{5 7 , 5 0 \%}$ \\
\hline
\end{tabular}


Tabela 3: Serviços onde os endodontistas atuam em números absolutos e em porcentagem

\begin{tabular}{lcc}
$\begin{array}{c}\text { Serviços onde os entrevistados } \\
\text { atuam }\end{array}$ & $\begin{array}{c}\text { Número } \\
\text { absoluto } \\
44\end{array}$ & $\begin{array}{c}\text { Número } \\
\text { em \% }\end{array}$ \\
Serviço público & $\begin{array}{c}44 \\
\text { pessoas }\end{array}$ & $60,20 \%$ \\
Consultório particular & $\begin{array}{c}71 \\
\text { pessoas } \\
11\end{array}$ & $97,20 \%$ \\
Empresa Privada & pessoas & $15,00 \%$ \\
Planos de Saúde & 04 & \\
\hline
\end{tabular}

Tabela 5: Endodontistas que Realizam o desbridamento foraminal em números absolutos e em porcentagem

\begin{tabular}{clc}
$\begin{array}{c}\text { Realizam o desbridamento } \\
\text { foraminal }\end{array}$ & $\begin{array}{l}\text { Número } \\
\text { absoluto }\end{array}$ & $\begin{array}{c}\text { Número } \\
\text { em \% }\end{array}$ \\
& $\begin{array}{l}71 \\
\text { pessoas }\end{array}$ & $97,30 \%$ \\
& 02 & \\
& pessoas & $2,70 \%$ \\
\hline
\end{tabular}

Tabela 6: Situações onde os entrevistados realizam o desbridamento foraminal em números absolutos e em porcentagem

$\begin{array}{ccc}\begin{array}{c}\text { Situações onde os entrevistados } \\ \text { realizam o desbridamento } \\ \text { foraminal }\end{array} & \begin{array}{c}\text { Número } \\ \text { absoluto }\end{array} & \begin{array}{c}\text { Número } \\ \text { em } \%\end{array}\end{array}$

\begin{tabular}{lcc}
$\begin{array}{l}\text { Em todos os tratamentos } \\
\text { endodônticos }\end{array}$ & $\begin{array}{c}18 \\
\text { pessoas } \\
\end{array}$ & $25,30 \%$ \\
Em todos os casos de Necrose & pessoas & $54,90 \%$ \\
& 02 & \\
Apenas em Necrose sem lesão & pessoas & $2,80 \%$ \\
& 12 \\
Apenas em Necrose com lesão & pessoas & $16,90 \%$ \\
\hline
\end{tabular}

Tabela 7: Momento em que os entrevistados realizam 0 desbridamento foraminal em números absolutos e em porcentagem

\begin{tabular}{ccc}
$\begin{array}{c}\text { Momento em que os entrevistados } \\
\text { realizam o desbridamento } \\
\text { foraminal }\end{array}$ & $\begin{array}{c}\text { Número } \\
\text { absoluto } \\
\text { Antes do preparo biomecânico }\end{array}$ & $\begin{array}{c}\text { Número } \\
\text { em } \%\end{array}$ \\
pessoas & $8,40 \%$ \\
Durante o preparo biomecânico & 51 & \\
& pessoas & $71,80 \%$ \\
Após o preparo biomecânico & 22 & \\
\hline
\end{tabular}

\section{Discussão}

Um planejamento minucioso do caso, antes de iniciar a micro cirurgia no interior da câmara pulpar, representada pelo preparo químico mecânico do sistema de canais radiculares, é essencial para que o profissional não seja surpreendido durante sua execução.

O canal radicular termina no forame, porem nem sempre está localizado na extremidade apical da raiz, em mais de $60 \%$ dos casos não está localizado no ápice radiográfico, podendo ser essa distância de 0 a 3mm, lateralmente (MARTOS;CASTRO; GONZÁLEZ-RODRÍGUEZ; FERRER-LUQUE, 2009).

Entretanto o limite apical de instrumentação em dentes com polpas vital e necróticas ainda é uma fonte de discussão e controvérsia na Endodontia, sendo defendido por alguns autores que o limite de instrumentação fique situado entre 0 e $1 \mathrm{~mm}$ milímetro aquém do forame (SIQUEIRA JR; RÔÇAS; LOPES, et al., 2011).

O objetivo final do preparo biomecânico é eliminar as bactérias do sistema de canais radiculares. A capacidade de limpar completamente e modelar as complexidades anatômicas do sistema de canais é o principal determinante para o sucesso endodôntico (ELEMAM; PRETTY, 2011)

Mais recentemente, a patência tem sido preconizada como um procedimento realizado durante a instrumentação que objetiva a recapitulação a fim de não perder o comprimento de trabalho, associado ao conceito de limpeza do forame, no qual uma lima de maior calibre deve ser usada (RIBEIRO; MALNATI; COSTA JÚNIOR, 2010). Dessa forma, esse procedimento está bem indicado somente em casos de necrose pulpar.

Neste estudo foi utilizado um questionário de auto-relato para obter informações de endodontistas sobre as suas atitudes e protocolos de realização do desbridamento foraminal durante a terapia endodôntica. A taxa de resposta conseguida permite uma avaliação válida. Este método é amplamente utilizado em modelos transversais de estudo onde todos os dados podem ser recolhidos a qualquer tempo, sem interferência do entrevistador. Além disso, a confidencialidade dos questionários permitiu maior confiança nas respostas e pode contribuir para a alta taxa de resposta.

O ápice radicular pode reter microrganismos com potencial para causar inflamação perirradicular, muitas vezes exacerbadas com dor, edema e/ou exsudação, portanto, as intervenções de tratamento que promovam a remoção desses agentes patogênicos devem ser indicadas no tratamento de sistemas de raízes infectadas canal (MITTAL, 2010).

A microflora apical polimicrobiana, com maior prevalência de estreptococos e microorganismos aeróbios pode desempenhar um papel significativo nas falhas do tratamento endodôntico. $O$ reconhecimento de tal situação tem contribuído para difundir a aceitação da limpeza e desbridamento do forame apical durante a instrumentação do canal radicular e esse entendimento reflete mudanças importantes na prática rotineira dos endodontistas (SOUZA, 2006). Isto é nitidamente percebido nesta pesquisa pelo elevado número de profissionais que afirmam realizar tal procedimento em seus protocolos clínicos.

O desbridamento do forame apical é um passo relevante para a endodontia atual, sobretudo pela proposta de limpeza foraminal com diminuição ou eliminação do acúmulo de raspas de dentina que pode levar a ocorrência dos temidos flare ups e comprometer o reparo do terço apical (EHRMANN; MESSER; CLARK, 2007).

Mesmo promovendo pequena variação no diâmetro do forame apical, é uma manobra importante, sobretudo em canais infectados, pois interfere na flora bacteriana apical, induzindo uma resposta biológica positiva. (TINAZ; ALACAM; UZUN et al.; 2005; BAUGH; WALLACE, 2005). 
O tempo de formado não representou um diferencial significativo na decisão para realizar o procedimento, no entanto percebe-se que os profissionais que concluíram a especialização há menos de 10 apresentaram um percentual maior entre os que responderam o questionário, concordando com os relatos da literatura nesse período quando o tema passou a ser abordado com maior ênfase.

Apesar de ser um procedimento reconhecidamente eficaz na diminuição do biofilme bacteriano na região apical e responsável por elevar os índices de sucesso da terapia endodôntica, ainda não é consenso o melhor momento para a sua realização. Isso se torna evidente ao observarmos que a maioria dos entrevistados realiza o procedimento durante o preparo biomecânico, entretanto uma parcela significativa afirma realizá-lo antes e depois do preparo.

$O$ reconhecimento da presença de microrganismos no canal cementário, e em lesões periapicais, tem contribuído para difundir a aceitação da limpeza e desbridamento do forame apical durante a instrumentação do canal radicular. No entanto, a possível existência de um tronco de polpa vital em casos de necrose pulpar sem lesão periapical tem impedido a aceitação integral desses procedimentos por endodontistas e pesquisadores (SOUZA, 2006). Observação concordante com os nossos resultados onde 2,8\% dos entrevistados afirmaram apenas realizar o procedimento nos casos de necrose sem lesão periapical visível radiograficamente. Entretanto de maneira controversa, apesar de a literatura mostrar que em casos de polpa viva não se faz desbridamento, com o intuito de preservar o coto pulpar, $25 \%$ dos entrevistados o realizam mesmo em polpas com vitalidade.

\section{Conclusão}

Os resultados desta pesquisa sugerem que 0 desbridamento foraminal está consolidado nos protocolos endodônticos dos especialistas do Estado de Pernambuco.

\section{Referências}

BAUGH. D.; WALLACE, J. The Role of Apical Instrumentation in Root Canal Treatment: A Review of the Literature. JOE, vol. 31, no. 5, p. 333-340, 2005.

CAMOES, I.C.G.; SALLES, M.R.; FERNANDO, M.V.M.; FREITAS, L.F.; GOMES, C.C. Relationship between the size of patency file and apical extrusion of sodium hypochlorite. IJDR, vol. 20, no. 4, p. 426430, 2009.

ELEMAM, R.F.; PRETTY, I. Comparison of the Success Rate of Endodontic Treatment and Implant Treatment, ISRN Dentistry, vol. 2011, 2011.

EHRMANN, E. H.; MESSER, H.H.; CLARK, R.M. Flare-ups in endodontics and their relationship to various medicaments. Aust Endod J., vol. 33, no. 3, p. 119-130, 2007.

GUIMARÃES, C.S. Influência da limpeza do forame apical na regressão de lesões periapicais. Monografia (Especialização em Endodontia). Associação Brasileira de Odontologia - Seção Pernambuco, Recife, 35 páginas, 2006
GUIMARÃES, C.S.; SILVA, L.B.; SANTOS, R.A. Foramen cleaning: a critical analysis. Internet J. Dental Science, [S.I.], vol. 7, no. 2, p. 13, Oct. 2009.

LEONARDI, L.E.; ATLAS, D.M.; RAINDEN, G. Apical extrusion of debris by manual and mechanical instrumentation. Braz Dent J., vol. 18, no. , p. 16-19, 2007.

MARTOS, J.; FERRER-LUQUE, C. M.; GONZÁLEZ-RODRÍGUEZ, M. P.;CASTRO, L. A. S. Topographical evaluation of the major apical foramen in permanent human teeth. International Endodontic Journal., vol. 42, p. 329-334, 2009.

MITTAL, R. Endodontic Flare - Ups: An Overview. J Oral Health Comm Dent., vol. 4, no. 3, p.67-71, 2010.

RIBEIRO, A.P.D.; MALNATI, P.S.; COSTA JÚNIOR, E.D. Limpeza do forame e extrusão apical de raspas de dentina em dentes unirradiculares submetidos ao procedimento de patência apical. Pesq. Bras. Odontoped. Clin. Integr., João Pessoa, vol. 10, no. 1, p. 55-60, jan./abr. 2010.

SCHAEFFER, M.A.; WHITE, R.R.; WALTON, R.E. Determining the optimal obturation length: a meta-analysis of literature. J Endod., vol. 31, p. 271-274, 2005.

SILVA, J.M.; CRUZ, H.M.; ARAÚJO, L.M.; PESSOA, O.F. The evaluation of removal of calcium hydroxide using different irrigation methods. Rev Odontol UNESP, vol.38, no.1, p. 37-43, 2009.

SILVA, L.B.; GUIMARAES C.S.; SILVEIRA M.M.F. Single and multiple session endodontic treatment of teeth with periapical diseases: a case report. The Internet Journal of Dental Science., vol.7, no. 2, 2009.

SIQUEIRA JR, J.F.; RÔÇAS, I.N.; LOPES, H.P.; ALVES, F. R. F.; OLIVEIRA, J.C.M.; ARMADA, L. Biological principles of endodontic treatment of teeth with vital pulp. Rev. bras. odontol., Rio de Janeiro, vol. 68 , no. 2, p. 161-165, 2011.

SOUZA, R.A. The importance of apical patency and cleaning of the apical foramen on root canal preparation. Braz. Dent. J., vol.17, no.1, p, 6-9, 2006.

TINAZ, A.C.; ALACAM, T.; UZUN, O.; MADEN, M.; KAYAOGLU, G. The effect of disruption of apical constriction on periapical extrusion. $\mathbf{J}$ Endod., vol. 31, no. 7, p. 533-535, 2005. 